\title{
Aspects of the cosmic microwave background dipole
}

\author{
Marc Kamionkowski \\ Mail Code 130-33, California Institute of Technology, Pasadena, California 91125 \\ Lloyd Knox \\ Department of Physics, University of California, Davis, California 95616
}

(Received 1 November 2002; published 17 March 2003)

\begin{abstract}
Cosmic microwave background $(\mathrm{CMB})$ experiments generally infer a temperature fluctuation from a measured intensity fluctuation through the first term in the Taylor expansion of the Planck function, the relation between the intensity in a given frequency and the temperature. However, with the forthcoming Planck satellite, and perhaps even with the Microwave Anisotropy Probe, the CMB-dipole amplitude will be large enough to warrant inclusion of the next higher order term. To quadratic order in the dipole amplitude, there is an intensity quadrupole induced by the dipole with a frequency dependence given by the second derivative of the Planck function. The Planck satellite should be able to detect this dipole-induced intensity quadrupole and distinguish it through its frequency dependence from the intrinsic CMB temperature and foreground quadrupoles. This higher-order effect provides a robust pre-determined target that may provide tests of Planck's and MAP's large-angle-fluctuation measurements and of their techniques for multifrequency foreground subtraction.
\end{abstract}

DOI: 10.1103/PhysRevD.67.063001

\section{INTRODUCTION}

The primary aim of NASA's recently launched Microwave Anisotropy Probe (MAP) [1] and the European Space Agency's Planck satellite [2] will be to measure small-scale fluctuations in the cosmic microwave background (CMB) in order to better determine cosmological parameters $[3,4]$ and test inflationary cosmology [5], and thus improve upon the already remarkable results from recent balloon and ground experiments [6]. However, the most salient feature in the CMB is not this small-scale structure; it is the much more prominent dipole [7] of amplitude $\Delta T=3.365 \pm 0.027 \mathrm{mK}$.

The simplest explanation for the dipole is a local group velocity $v=627 \pm 22 \mathrm{~km} \mathrm{sec}^{-1}$ toward $(l, b)=\left(276^{\circ}\right.$ $\pm 3^{\circ}, 33^{\circ} \pm 3^{\circ}$ ) with respect to the CMB rest frame. Some measurements of galaxy velocities on large scales suggest independently, although far less precisely, a similar value [8]. It has thus been generally accepted that the CMB dipole is due to our peculiar velocity, but theorists have occasionally speculated that the dipole might be due, at least in part, to an intrinsic temperature fluctuation [9].

If we had sufficiently precise multi-frequency observations to measure the frequency spectrum at each point on the sky, it would always be consistent with a blackbody spectrum, although with an angle-dependent temperature. In practice, the observation frequencies and sensitivities are limited. The temperature at each point on the sky is thus determined by measuring the intensity fluctuation, and then converting it to a temperature fluctuation, generally assuming that the temperature fluctuation is sufficiently small that the intensity fluctuation can be related to the temperature fluctuation by the first term in a Taylor expansion of the Planck function, the relation between the temperature and intensity. With prior experiments, the temperature fluctuation has always been sufficiently small that this approximation has been warranted.

In this paper we point out that with the observed dipole
PACS number(s): $98.70 . \mathrm{Vc}$

amplitude and Planck's (and possibly MAP's) sensitivity, this will no longer be a good approximation-the second-order term in the Taylor expansion will be non-negligible. To quadratic order in the dipole amplitude, there is an intensity quadrupole with a frequency dependence given by the second derivative of a Planck function. Since this frequency dependence differs from that of the first-order term, this dipole-induced intensity quadrupole can be disentangled from the temperature quadrupole, even after taking into account a number of foregrounds, as we show below. This higher-order effect will provide a robust pre-determined signal that Planck and possibly MAP should be able to detect. It can be used to calibrate large-angle-fluctuation measurements and/or benchmark multi-frequency foregroundsubtraction techniques. It may thus be a useful addition to the astrophysical point sources and annual modulation of the dipole that have until now served as calibration sources for CMB experiments.

In the next section we discuss the dipole-induced intensity quadrupole, which we refer to simply as the "dipole quadrupole" (DQ). In Sec. III, we then show that the DQ should be detectable by Planck and distinguishable from the CMB quadrupole, even after subtracting several foregrounds. Our analysis in Secs. II and III assumes that the dipole is due entirely to the velocity of the solar system with respect to the CMB rest frame. To indicate the magnitude and detectability of the DQ, we evaluate how well the magnitude and orientation of the peculiar velocity could be determinedassuming the dipole were due to a peculiar velocity-from the DQ alone. In Sec. IV we show that the same DQ arises even if the CMB dipole is an intrinsic temperature fluctuation, rather than the result of a peculiar velocity. Section V provides some closing remarks.

\section{THE DIPOLE-INDUCED INTENSITY QUADRUPOLE} is 


$$
I_{\nu^{\prime}}^{\prime}=C \frac{x^{\prime 3}}{e^{x^{\prime}}-1},
$$

where $\quad C=2\left(k_{B} T_{\mathrm{CMB}}\right)^{3}(h c)^{-2}$ is a constant, $x^{\prime}$ $=h \nu^{\prime}\left(k_{B} T_{\mathrm{CMB}}\right)^{-1}, \nu^{\prime}$ is the photon frequency, and $h, k_{B}$, and $c$ are, respectively, the Planck and Boltzmann constants and the speed of light. The photon frequency measured by an observer moving with velocity $v$ relative to the blackbody is $\nu=\gamma \nu^{\prime}(1+\beta \mu)$, where $\beta=v / c, \gamma=\left(1-\beta^{2}\right)^{-1 / 2}$, and $\mu$ is the cosine of the angle between the velocity and the photon direction. The specific intensity transforms as $I_{\nu^{\prime}}^{\prime} / \nu^{\prime 3}$ $=I_{\nu} / \nu^{3}$, and thus the observer-frame specific intensity is

$$
I_{\nu}=C \frac{x^{3}}{e^{x \gamma(1+\beta \mu)}-1},
$$

where $x=h \nu\left(k_{B} T_{\mathrm{CMB}}\right)^{-1}$. Expanding in $\beta$,

$$
\begin{aligned}
I_{\nu}= & C \frac{x^{3}}{e^{x}-1}\left\{\left[1+O\left(\beta^{2}\right)\right]-f(x) \beta \mu\right. \\
& \left.+f(x) g(x) \beta^{2}\left(\mu^{2}-1 / 3\right)+\cdots\right\},
\end{aligned}
$$

where $f(x) \equiv x e^{x}\left(e^{x}-1\right)$ and $g(x) \equiv(x / 2) \operatorname{coth}(x / 2)$.

The term linear in $\beta$ is the dipole, with the appropriate frequency spectrum $f(x)$ for the dipole [10], which is also more generally the frequency spectrum for a thermal fluctuation. However, to order $\beta^{2}$, there is another term, the DQ, with a frequency spectrum that differs from that from small thermal fluctuations. With COBE Differential Microwave Radiometer's (DMR's) sensitivity and frequencies (in the Rayleigh-Jeans regime), the frequency dependence was negligible [11]. However, with the improved sensitivity and expanded frequency range of new satellite experiments, the frequency dependence will become detectable. This frequency dependence was noted in Refs. [12], but seems to have since been overlooked. References [13] identified the effect (sometimes referred to as the "kinematic quadrupole") in calculations of CMB polarization induced by reionization, but they did not consider the CMB dipole. Reference [14] discusses peculiar-velocity effects on the CMB, but only on smallscale fluctuations.

\section{DETECTABILITY OF THE DQ}

To assess the detectability of the DQ, we determine how well the amplitude and direction of the solar-system velocity can be determined with MAP and Planck under the assumption that the velocity is to be reconstructed entirely from the DQ.

Since the frequency dependences of the CMB quadrupole and the DQ differ, they can be distinguished if the quadrupole moments of the intensity are determined in several frequencies. However, the CMB quadrupole and the DQ will also have to be distinguished from the quadrupole moments due to unsubtracted, or imperfectly subtracted, foregrounds, each with its own frequency dependence.

In what follows we discuss the brightness temperature (which is proportional to the intensity) rather than the inten-

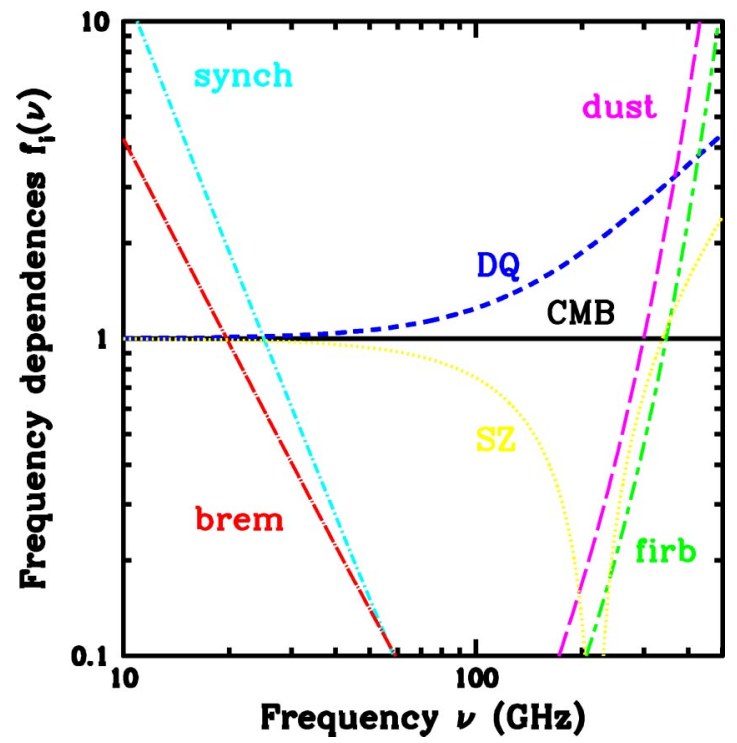

FIG. 1. The frequency dependence of the cosmological (solid line), kinematic (short-dash line), dust (long-dash line), synchrotron (dot-short-dash line), bremsstrahlung (dot-long-dash line), FIRB (short-dash-long-dash line), and Sunyaev-Zeldovich quadrupole moments, all referenced to the frequency dependence of the cosmological quadrupole. For the Sunyaev-Zeldovich effect, we plot the absolute value as the frequency dependence changes sign near $\nu$ $=220 \mathrm{GHz}$. The amplitudes are arbitrary, as our analysis assumes that they will be determined by the data.

sity so that we work with units that are familiar in CMB studies. If the brightness temperature $T_{\nu}(\hat{\mathbf{n}})$ is measured in several frequencies $\nu$ as a function of position $\hat{\mathbf{n}}$ over the entire sky, then the five (for $m=-2,-1,0,1,2$ ) quadrupole components $T_{m, \nu}$ can be constructed from $T_{m, \nu}$ $=\int Y_{2 m}(\hat{\mathbf{n}}) T_{\nu}(\hat{\mathbf{n}}) d \hat{\mathbf{n}}$, where $Y_{l m}(\hat{\mathbf{n}})$ are spherical harmonics. The possible contributions to these $T_{m, \nu}$ from several unsubtracted foregrounds [dust, synchrotron radiation, bremsstrahlung, the far infrared background (FIRB) and the SunyaevZeldovich effect], which will have frequency dependences shown in Fig. 1, must also be considered. The quadrupole moment at each frequency is then the sum of the contributions from all of these sources: $T_{m, \nu}=\Sigma_{i} T_{m}^{i} f_{i}(\nu)$, where $i$ $=\{\mathrm{CMB}, \mathrm{DQ}$, dust,synch,brem,firb,SZ $\}$, and $f_{i}(\nu)$ is the frequency dependence of source $i$, referenced to the CMB frequency dependence. Thus, $f_{\mathrm{CMB}}(\nu)=1 ; \quad f_{\mathrm{DQ}}(\nu)$ $=(x / 2) \operatorname{coth}(x / 2) ; \quad f_{\text {dust }}(\nu)=x^{2} e^{x_{d}-x}\left(e^{x}-1\right)^{2}\left(e^{x_{d}-1}\right)^{-2}$ [where $x_{d}=h \nu\left(k_{B} T_{\text {dust }}\right)^{-1}$ and we have taken a thermal dust spectrum with $T_{\text {dust }}=20 \mathrm{~K}$ and emissivity index $\alpha=2$ ]; $f_{\text {synch }}(\nu)=x^{-4.8}\left(e^{x}-1\right)^{2} e^{-x} ; f_{\text {brem }}(\nu)=x^{-4.16}\left(e^{x}-1\right)^{2} e^{-x}$; $f_{\text {firb }}(\nu)=x^{1.64} e^{x_{f}-x}\left(e^{x}-1\right)^{2}\left(e^{\left.x_{f}-1\right)^{-2}} \quad\right.$ [where $x_{f}$ $=h \nu\left(k_{B} T_{\text {firb }}\right)^{-1}$ and we have taken a thermal FIRB spectrum with $T_{\text {firb }}=18.5 \mathrm{~K}$ and emissivity index $\alpha=0.64$ as a fit to data [15]]; and $f_{\mathrm{SZ}}(\nu)=2 f_{\mathrm{CMB}}(\nu)-f_{\mathrm{DQ}}(\nu)$. For a review of foreground properties see Ref. [16].

For each of the five components $m$, we will have five (for the MAP frequencies of 22, 30, 40,60, and $90 \mathrm{GHz}$ ) or nine (for the Planck frequencies of 30, 44, 65, 100, 143, 217, 353, 545 , and $857 \mathrm{GHz}$ ) data points $T_{m, \nu}$ that we model with a vector of parameters $s_{i}=T_{m}^{i}$ again for $i$ 
$=\{\mathrm{CMB}, \mathrm{DQ}$,dust,synch,brem,firb,SZ $\}$. Following Ref. [4], the standard error $(1 \sigma)$ to the quadrupole component $T_{m}^{\mathrm{DQ}}$ will be $\sigma_{m}^{\mathrm{DQ}}=\left[\alpha^{-1 / 2}\right]_{\mathrm{DQ}, \mathrm{DQ}}$, where

$$
\alpha_{i j}=\sum_{\nu}\left[\frac{\partial T_{m, \nu}}{\partial s_{i}} \frac{\partial T_{m, \nu}}{\partial s_{j}}\right] \frac{1}{\sigma_{m, \nu}^{2}}=\sum_{\nu} \frac{f_{i}(\nu) f_{j}(\nu)}{\sigma_{m, \nu}^{2}},
$$

is the covariance (Fisher) matrix. Here, $\sigma_{m, \nu}^{2}$ is the variance to the quadrupole from instrumental noise at frequency $\nu$; we use 4-year values for MAP from Ref. [1] and 2-year values for Planck from Ref. [2].

Once the DQ components $T_{m}^{\mathrm{DQ}}$ have been determined, the peculiar-velocity components can be found. Expanding the angular dependence, $\left(\mu^{2}-1 / 3\right)$, where $\mu$ is the cosine of the angle between the velocity and the photon direction, in spherical harmonics gives us

$$
\begin{aligned}
\left(T_{m=0}^{\mathrm{DQ}} / T_{\mathrm{CMB}}\right) & =-\sqrt{4 \pi / 45}\left(\beta_{x}^{2}+\beta_{y}^{2}-2 \beta_{z}^{2}\right), \\
\sqrt{2}\left(\operatorname{Re} T_{m=1}^{\mathrm{DQ}} / T_{\mathrm{CMB}}\right) & =-\sqrt{16 \pi / 15} \beta_{x} \beta_{z}, \\
\sqrt{2}\left(\operatorname{Im} T_{m=1}^{\mathrm{DQ}} / T_{\mathrm{CMB}}\right) & =-\sqrt{16 \pi / 15} \beta_{y} \beta_{z}, \\
\sqrt{2}\left(\operatorname{Re} T_{m=2}^{\mathrm{DQ}} / T_{\mathrm{CMB}}\right) & =-\sqrt{4 \pi / 15}\left(\beta_{x}^{2}-\beta_{y}^{2}\right),
\end{aligned}
$$

and

$$
\sqrt{2}\left(\operatorname{Im} T_{m=2}^{\mathrm{DQ}} / T_{\mathrm{CMB}}\right)=-\sqrt{16 \pi / 15} \beta_{x} \beta_{y} .
$$

Let us suppose that these moments have been measured precisely. Then the equations for $T_{m=0}^{\mathrm{DQ}}(\equiv a), \operatorname{Re} T_{m=2}^{\mathrm{DQ}}(\equiv b)$, and $\operatorname{Im} T_{m=2}^{\mathrm{DQ}}(\equiv c)$ can be inverted (after introducing the shorthand $a, b$, and $c$ ) to give us the components

$$
\begin{aligned}
& \beta_{x}^{2}=\sqrt{15(16 \pi)^{-1}}\left(\sqrt{b^{2}+c^{2}}+b\right), \\
& \beta_{y}^{2}=\sqrt{15(16 \pi)^{-1}}\left(\sqrt{b^{2}+c^{2}}-b\right),
\end{aligned}
$$

and

$$
\beta_{z}^{2}=\sqrt{15(16 \pi)^{-1}}\left(\sqrt{b^{2}+c^{2}}-\sqrt{3} a\right) .
$$

Since we have now determined the squares of three components of the velocity, we are still left with a residual eightfold degeneracy in the velocity. However, the signs of the components $\operatorname{Re} T_{m=1}^{\mathrm{DQ}}$ and $\operatorname{Im} T_{m=1}^{\mathrm{DQ}}$, which are proportional, respectively, to $\beta_{x} \beta_{z}$ and $\beta_{y} \beta_{z}$, can be used to break two of these degeneracies, leaving us with only a twofold degeneracy, the sign of the velocity, which is undetermined by the quadrupole.

When the measurements are done, we will then want to check whether the peculiar velocity induced from the DQ is consistent with that inferred from the dipole itself. If the data analysis and our understanding of the instrument are reliable, then the two velocities should be consistent. To quantify the degree to which MAP and Planck can test this consistency we calculate, assuming $v=0$, the expectation value of $\chi^{2}$ for the hypothesis that $v=627 \mathrm{~km} \mathrm{sec}^{-1}$. If, e.g., $\chi^{2}=9$ then the $v=0$ hypothesis can be distinguished from the $v$
$=627 \mathrm{~km} \mathrm{sec}^{-1}$ hypothesis at $3 \sigma$. We find, for arbitrary $v$, $\chi^{2}=9\left(4.2 \mu \mathrm{K} / \sigma_{m}^{\mathrm{DQ}}\right)^{2}\left[v /\left(627 \mathrm{~km} \mathrm{sec}^{-1}\right)\right]^{4}$. From this $\chi^{2}$ we also derive the minimum $v$ distinguishable at $3 \sigma$ from $v=0$ to be $v_{\min }=305 \mathrm{~km} \mathrm{sec}^{-1}\left(\sigma_{m}^{\mathrm{DQ}} / \mu \mathrm{K}\right)^{1 / 2}$.

As another indicator of the precision with which the DQ can be reconstructed, we now determine the precision with which the magnitude and orientation of the velocity can be inferred from the DQ alone. There will be a measurement error of $\sigma_{m}^{\mathrm{DQ}}$ to each of the $T_{m}^{\mathrm{DQ}}$, as described above, and the three velocity components, $v_{x}, v_{y}$, and $v_{z}$, will be fit to all five of these quadrupole components. To estimate the errors on these components we once again calculate a Fisher matrix, this time for these five data points and the parameters $v_{x}, v_{y}$, and $v_{z}$. We then invert the Fisher matrix and choose the $z$ axis along the inferred velocity so that $v=v_{z}$. We then find that the measurement error (at $3 \sigma$ ) to the velocity is $\Delta v=3 \sqrt{45(64 \pi)^{-1}} \sigma_{m}^{\mathrm{DQ}} c^{2}\left(v_{z} T_{\mathrm{CMB}}\right)^{-1}$, where $v$ is the bestfit velocity. Under the null hypothesis that the peculiar velocity is that inferred from the dipole, we find $\Delta v$ $=225\left(\sigma_{m}^{\mathrm{DQ}} / \mu \mathrm{K}\right) \mathrm{km} \mathrm{sec}^{-1}$. The Fisher-matrix analysis tells us that the measurement errors to the $x$ and $y$ components (those perpendicular to the best-fit velocity) are each $\sqrt{4 / 3}$ times that for the $z$ component. Thus, under the same null hypothesis, the $(3 \sigma)$ error to the velocity orientation will be $24^{\circ}\left(\sigma_{m}^{\mathrm{DQ}} / \mu \mathrm{K}\right)$.

Table I shows results of our calculations of $\chi^{2}$ and $\Delta v$ assuming a variety of combinations of components will be fit to the data. If the dust contribution to MAP must be determined from the MAP data itself, then the smallest detectable peculiar velocity is $\sim 2100 \mathrm{~km} \mathrm{sec}^{-1}$, too big to be interesting. This is because the highest-frequency channel becomes a dust monitor, and the remaining MAP channels at $\$ 60 \mathrm{GHz}$ do not provide enough leverage to disentangle the DQ and the CMB quadrupole. However, if the dust quadrupole can be determined precisely from other observations, then MAP might be able to isolate the DQ to better than $7 \sigma$. The synchrotron and bremsstrahlung foregrounds are strongest at low frequencies, where the DQ and CMB frequency dependences are similar. Thus, if synchrotron and bremsstrahlung emission are included in the analysis, the lowestfrequency channels become foreground monitors and the ability to separate the DQ is not degraded significantly.

Because of the improved detector sensitivity, and especially the broadened frequency coverage, the outlook for Planck is much better. Even without the highest-frequency channels, which act as dust monitors, there is still a sufficiently broad spectrum of frequencies $\gtrsim 100 \mathrm{GHz}$ where the spectral dependences of the DQ and the CMB quadrupole differ the most. Again, the synchrotron and bremsstrahlung foregrounds contribute mostly at low frequencies and thus do not degrade significantly the DQ signal. Quite remarkably, even if we marginalize over a number of uncertain foreground amplitudes, Planck should be able to detect the DQ as long as the velocity is greater than roughly $140 \mathrm{~km} \mathrm{sec}^{-1}$. Moreover, Planck should be able to detect a deviation from the dipole-inferred velocity as small as $45 \mathrm{~km} \mathrm{sec}^{-1}$ and determine its direction (modulo the sign) to better than $5^{\circ}$. 
TABLE I. Values for detectability of the dipole-induced intensity quadrupole. The $\sigma_{m}^{\mathrm{DQ}}$ column gives the standard error to the DQ amplitude. The $\chi^{2}$ column quantifies the ability to distinguish $v=0$ from $v$ $=627 \mathrm{~km} \mathrm{sec}^{-1}$ using the DQ. The quadrupole components that are assumed to be fit to the multifrequency data are the DQ, CMB quadrupole (T), dust (D), synchrotron radiation (Synch), bremsstrahlung (Brem), far infrared background (FIRB), and Sunyaev-Zeldovich effect (SZ). The "NA" in the $\chi^{2}$ column indicates that if the SZ effect is taken into account, our covariance matrix formally gives an infinite result because of the degeneracy between the frequency dependences of the CMB, the DQ, and Sunyaev-Zeldovich effect. The column $\Delta v$ gives the error (at $3 \sigma$ ) to the magnitude of the velocity. We list NA for $\Delta v$ for the cases where the DQ is unlikely to be detected. The last column gives the anticipated $(3 \sigma)$ error to the orientation of the velocity.

\begin{tabular}{|c|c|c|c|c|c|}
\hline Experiment & Components & $\sigma_{m}^{\mathrm{DQ}}(\mu \mathrm{K})$ & $\chi^{2}$ & $\Delta v\left(\mathrm{~km} \mathrm{sec}^{-1}\right)$ & $\Delta \theta(\mathrm{deg})$ \\
\hline MAP & DQ & 0.04 & $10^{5}$ & 9 & 0.9 \\
\hline MAP & $\mathrm{DQ}+\mathrm{T}$ & 0.6 & 440 & 140 & 14 \\
\hline MAP & $\mathrm{DQ}+\mathrm{T}+$ Synch + Brem & 1.7 & 52 & 390 & 42 \\
\hline MAP & $\mathrm{DQ}+\mathrm{T}+\mathrm{D}$ & 16 & 0.66 & NA & NA \\
\hline MAP & $\mathrm{DQ}+\mathrm{T}+\mathrm{D}+$ Synch + Brem & 73 & 0.032 & NA & NA \\
\hline Planck & DQ & 0.005 & $6 \times 10^{6}$ & 1.1 & 0.12 \\
\hline Planck & $\mathrm{DQ}+\mathrm{T}$ & 0.02 & $3 \times 10^{5}$ & 4.9 & 0.52 \\
\hline Planck & $\mathrm{DQ}+\mathrm{T}+\mathrm{D}$ & 0.02 & $3 \times 10^{5}$ & 5.0 & 0.53 \\
\hline Planck & $\mathrm{DQ}+\mathrm{T}+\mathrm{D}+\mathrm{FIRB}$ & 0.036 & $10^{5}$ & 8.1 & 0.86 \\
\hline Planck & $\mathrm{DQ}+\mathrm{T}+\mathrm{D}+\mathrm{FIRB}+\mathrm{SZ}$ & NA & $\mathrm{A}$ & NA & NA \\
\hline Planck & $\mathrm{DQ}+\mathrm{T}+\mathrm{D}+\mathrm{FIRB}+$ Synch + Brem & 0.2 & 4,000 & 45 & 4.8 \\
\hline
\end{tabular}

It turns out that the Sunyaev-Zeldovich frequency dependence is a linear combination of the CMB-quadrupole and DQ frequency dependences. Thus, the DQ is formally degenerate with the cosmological and Sunyaev-Zeldovich quadrupoles. However, the quadrupole moment due to the SunyaevZeldovich effect will probably be negligible. Calculations of the Sunyaev-Zeldovich power spectrum near $l \simeq 100$ find $l^{2} C_{l} /(2 \pi) \lesssim 10^{-12}$ [17]. Doing shot-noise extrapolation to the quadrupole, we find $\sigma_{m}^{\mathrm{SZ}} \leq 0.15 \mu \mathrm{K}$, corresponding to a velocity uncertainty (at $3 \sigma$ ) $\$ 35 \mathrm{~km} \mathrm{sec}^{-1}$, which is just a bit below the expected statistical uncertainty indicated in Table I for Planck. The actual SZ number will probably be much smaller, and certainly much more will be known by the time Planck flies. The SZ octupole will be useful in constraining the possible SZ quadrupole contribution.

Our foreground modeling makes several simplifying assumptions. For example, we do not actually know the frequency dependences perfectly and they can vary spatially (due to, e.g., spatial variation in chemical composition of the dust). There may also be components we have not yet considered, such as spinning dust. Further, about $25 \%$ of the sky will be lost to a galactic-plane cut. It is thus possible that Planck may not be able to achieve the tiny velocity errors quoted in the table. However, the values in the final row may be achievable. Although uncertain, the frequency dependences of the foregrounds are all (with the exception of the SZ effect) considerably different than those of the DQ and the CMB quadrupole. The foregrounds are all likely to have low amplitudes near $100 \mathrm{GHz}: \sim 3 \mu \mathrm{K}$ for dust, $\sim 1 \mu \mathrm{K}$ for bremsstrahlung and less for everything else [18]. Thus the DQ is not far below foreground quadrupoles, so multifrequency foreground subtraction need not be done to better than about $10 \%$. Of course, only the measurements themselves will answer these questions definitively.

\section{AN INTRINSIC DIPOLE?}

Although a peculiar velocity is the simplest explanation for the dipole, and certainly that most consistent with the prevailing inflationary paradigm, it is also possible that the dipole could be due, at least in part, to an intrinsic temperature dipole produced by a super-horizon entropy perturbation [9]. In this section, we show that even if the temperature dipole is intrinsic, the same DQ still arises. We also clarify the distinction between an intrinsic CMB temperature fluctuation, the thermal quadrupole induced by a peculiar velocity, and the DQ, which unlike the other two, is not a temperature quadrupole.

A velocity $v$ induces a temperature pattern $T(\theta)$ $=T_{\mathrm{CMB}}(1+\beta \mu)^{-1} \sqrt{1-\beta^{2}}[19]$. To second order in $\beta^{2}$, this can be written as

$$
T(\theta)=T_{\mathrm{CMB}}\left\{\left[1+O\left(\beta^{2}\right)\right]-\beta \mu+\beta^{2}\left(\mu^{2}-1 / 3\right)\right\} .
$$

Thus, the peculiar velocity induces a temperature quadrupole of magnitude $O\left(\beta^{2} T_{\mathrm{CMB}}\right)$, in addition to the dipole of amplitude $O\left(\beta T_{\mathrm{CMB}}\right)$. However, the quadrupole in the intensity [Eq. (3)] arises from a combination of both the temperature dipole and the temperature quadrupole. To see this, we rewrite Eq. (5) as

$$
T(\theta)=T_{\mathrm{CMB}}\left\{\left[1+O\left(\beta^{2}\right)\right]-\beta_{1} \mu+\beta_{2}^{2}\left(\mu^{2}-1 / 3\right)\right\},
$$

so that we can see where the DQ comes from. Doing so, we find that Eq. (3) becomes

$$
\begin{aligned}
I_{\nu}= & C \frac{x^{3}}{e^{x}-1}\left\{\left[1+O\left(\beta^{2}\right)\right]-f(x) \beta_{1} \mu+f(x)\right. \\
& \left.\times\left[\beta_{2}^{2}+\beta_{1}^{2}(g(x)-1)\right]\left(\mu^{2}-1 / 3\right)+\cdots\right\} .
\end{aligned}
$$


We thus see that what we have been calling the DQ consists of two parts: The first is the $\beta_{2}^{2}$ term, which is due to the temperature quadrupole induced by our peculiar velocity. Since this is an honest-to-goodness temperature quadrupole, it has the frequency dependence of the usual lowest-order thermal fluctuation, and it cannot be distinguished from an intrinsic $\mathrm{CMB}$ temperature fluctuation. The second term, proportional to $\beta_{1}^{2}$, arises from the term in the Taylor expansion of the intensity that is second order in the dipole amplitude. This second-order term has a frequency dependence that differs from the usual lowest-order thermal fluctuation.

It is also clear from Eqs. (6) and (7) that even if the temperature dipole were intrinsic (that is, due to an entropy perturbation), then there would still be a DQ. Although the frequency dependence is $g(x)-1$ rather than $g(x)$, only the part proportional to $g(x)$ can be distinguished by multifrequency maps from a temperature quadrupole. For this reason, the amplitude and orientation of the DQ would be exactly the same as if the dipole were due to a velocity, and the detectability would be exactly as determined above. Likewise, the DQ cannot be used to tell whether the dipole is due to a peculiar velocity or due to an entropy perturbation, as previously suggested [12]. ${ }^{1}$

Finally, we mention one last point. Strictly speaking, an intrinsic $\mathrm{CMB}$ temperature quadrupole will produce a contri-

${ }^{1}$ We thank E. Wright for illuminating discussions on this point and for pointing out an error in an earlier draft. bution to the intensity quadrupole with a frequency dependence proportional to $g(x)$, just like the DQ. However, if the temperature-quadrupole amplitude is $\Delta T\left(\sim 10^{-5}\right.$ for the intrinsic fluctuation and $\sim 10^{-6}$ for the velocity-induced temperature quadrupole), then this contribution will be of order $(\Delta T)^{2} \leq 10^{-10}$, much smaller than the $10^{-6}$ expected for the DQ.

\section{CONCLUSIONS}

We have shown that the dipole amplitude is sufficiently large that the discrepancy between the exact frequency dependence of a thermal fluctuation and the lowest-order frequency dependence usually assumed will be detectable by Planck and possibly MAP. To second-order in the dipole amplitude, this discrepancy is manifest as an intensity quadrupole that can be distinguished with multi-frequency measurements from an intrinsic temperature quadrupole. This provides a robust pre-determined target for CMB experiments and it may prove to be a useful tool for calibration for forthcoming space experiments.

\section{ACKNOWLEDGMENTS}

We thank the Santa Barbara KITP for hospitality. This work was supported at Caltech by NSF AST-0096023, NASA NAG5-9821, and DOE DE-FG03-92-ER40701, at Davis by NASA NAG5-11098, and at the KITP by NSF PHY99-07949.
[1] MAP Collaboration: http://map.gsfc.nasa.gov

[2] Planck Collaboration: http://sci.esa.int/planck

[3] L. Knox, Phys. Rev. D 52, 4307 (1995).

[4] G. Jungman, M. Kamionkowski, A. Kosowsky, and D.N. Spergel, Phys. Rev. D 54, 1332 (1996).

[5] For a review, see, e.g., M. Kamionkowski and A. Kosowsky, Annu. Rev. Nucl. Part. Sci. 49, 77 (1999).

[6] A.D. Miller et al., Astrophys. J. Lett. 524, L1 (1999); P. de Bernardis et al., Nature (London) 404, 955 (2000); S. Hanany et al., Astrophys. J. Lett. 545, L5 (2000); N.W. Halverson et al., Astrophys. J. 568, 38 (2002); B.S. Mason et al., astro-ph/0205384; A. Benoit et al., astro-ph/0210305; C.L. Kuo et al., astro-ph/0212289.

[7] E.K. Conklin, Nature (London) 222, 971 (1969); P.S. Henry, ibid. 231, 516 (1971); G.F. Smoot, M.V. Gorenstein, and R.A. Muller, Phys. Rev. Lett. 39, 898 (1977); E.S. Cheng, P.R. Saulson, D.T. Wilkinson, and B.E. Corey, Astrophys. J. Lett. 232, L139 (1979); P.M. Lubin, G.L. Epstein, and G.F. Smoot, Phys. Rev. Lett. 50, 616 (1983); D.J. Fixsen, E.S. Cheng, and D.T. Wilkinson, ibid. 50, 620 (1983); A. Kogut et al., Astrophys. J. 419, 1 (1994).

[8] A.G. Riess, W.H. Press, and R.P. Kirshner, Astrophys. J. Lett. 445, L91 (1995).

[9] L.P. Grishchuk and Ya.B. Zeldovich, Sov. Astron. 22, 125
(1978); D. Langlois, Phys. Rev. D 54, 2447 (1996); M.S. Turner, ibid. 44, 3737 (1991); B. Paczynski and T. Piran, Astrophys. J. 364, 341 (1990); D. Langlois and T. Piran, Phys. Rev. D 53, 2908 (1996); M. Jaroszynski and B. Paczynski, Astrophys. J. 448, 488 (1995).

[10] D.J. Fixsen et al., Astrophys. J. 420, 445 (1994).

[11] A. Kogut et al., Astrophys. J. Lett. 464, L5 (1996).

[12] P. de Bernardis et al., Astrophys. J. 353, 145 (1990); S. Bottani, P. de Bernardis, and F. Melchiorri, Astrophys. J. Lett. 384, L1 (1992).

[13] E. Audit and J.F.L. Simmons, Mon. Not. R. Astron. Soc. 305, L27 (1999); S.Y. Sazonov and R.A. Sunyaev, ibid. 310, 765 (1999); A.D. Challinor, M.T. Ford, and A.N. Lasenby, ibid. 312, 159 (2000); D. Baumann, A. Cooray, and M. Kamionkowski, astro-ph/0208511.

[14] A. Challinor and F. van Leeuwen, Phys. Rev. D 65, 103001 (2002).

[15] D.J. Fixsen et al., Astrophys. J. 508, 123 (1998).

[16] M. Tegmark, D.J. Eisenstein, W. Hu, and A. de Oliveira-Costa, Astrophys. J. 530, 133 (2000).

[17] V. Springel, M. White, and L. Hernquist, Astrophys. J. 549, 681 (2001)

[18] A. Kogut et al., Astrophys. J. 460, 1 (1996).

[19] P.J.E. Peebles and D.T. Wilkinson, Phys. Rev. 174, 2168 (1968). 